SUBJECT AREAS:

BIOINFORMATICS

CHEMINFORMATICS

Received

16 December 2013

Accepted

16 May 2014

Published

6 June 2014

Correspondence and requests for materials should be addressed to G.A. (archunan@bdu. ac.in) or P.P.

(ppadmanabhan 1@ gmail.com; ppadmanabhan@ntu. edu.sg)

* These authors contributed equally to this work.

\title{
Evaluating the binding efficiency of pheromone binding protein with its natural ligand using molecular docking and fluorescence analysis
}

\author{
Renganathan llayaraja ${ }^{*}$, Ramalingam Rajkumar ${ }^{2 *}$, Durairaj Rajesh', \\ Arumugam Ramachandran Muralidharan ${ }^{1}$, Parasuraman Padmanabhan ${ }^{3} \&$ Govindaraju Archunan ${ }^{1}$ \\ ${ }^{1}$ Centre for Pheromone Technology, Department of Animal Science, School of Life Sciences, Bharathidasan University, \\ Tiruchirappalli - 620 024, Tamil Nadu, India, ${ }^{2}$ Institute of Medical Biology, A-STAR, Biopolis Drive, Singapore, ${ }^{3}$ The Lee Kong \\ Chian School of Medicine, Nanyang Technological University, Singapore - 637553.
}

Chemosignals play a crucial role in social and sexual communication among inter- and intra-species. Chemical cues are bound with protein that is present in the pheromones irrespective of sex are commonly called as pheromone binding protein (PBP). In rats, the pheromone compounds are bound with low molecular lipocalin protein $\boldsymbol{\alpha}_{2 \mathrm{u}}$-globulin $(\boldsymbol{\alpha} 2 \mathrm{u})$. We reported farnesol is a natural endogenous ligand (compound) present in rat preputial gland as a bound volatile compound. In the present study, an attempt has been made through computational method to evaluating the binding efficiency of $\alpha 2 \mathrm{u}$ with the natural ligand (farnesol) and standard fluorescent molecule (2-naphthol). The docking analysis revealed that the binding energy of farnesol and 2-naphthol was almost equal and likely to share some binding pocket of protein. Further, to extrapolate the results generated through computational approach, the $\alpha 2 \mathrm{u}$ protein was purified and subjected to fluorescence titration and binding assay. The results showed that the farnesol is replaced by 2-naphthol with high hydrophobicity of TYR120 in binding sites of $\alpha 2 \mathrm{u}$ providing an acceptable dissociation constant indicating the binding efficiency of $\alpha 2 \mathrm{u}$. The obtained results are in corroboration with the data made through computational approach.

\footnotetext{
(2) hemical communication, a universal process of life, occurs at all levels of biological organization including the process of regulation of cells and organs within the body; they significantly influence the level of social behavior and ecological interactions among individuals ${ }^{1}$. The role of chemical molecules in mediating species-specific pheromonal communication is well documented in mammals including mouse ${ }^{2}$, rat $^{3}$, bovine $^{4}$, buffalo $^{5}$, and human ${ }^{6}$. The major functions of mammalian pheromones include sexual attraction, mounting ${ }^{7}$, mother-young interactions ${ }^{8}$, aggression and so on. Though a number of scent glands produce pheromones, the preputial gland is reported to be a major source of pheromone production ${ }^{9}$.

In mice, the pheromone binding protein synthesized in the liver and released into the blood plasma and excreted through urine are called to major urinary protein (MUPs) or pheromone binding protein (PBP), while its equivalent protein in rats is referred as $\alpha 2 \mathrm{u}^{2}$. This is also expressed in the urine and preputial gland of the rat. In male rats, the preputial glands are often better developed; in female similar gland is the clitoral gland. The preputial glandular activity has been documented through production of volatile compounds and sex associated proteins $(18 \mathrm{kDa}-\alpha 2 \mathrm{u})$ which are testosterone dependent ${ }^{10}$. Among the exhaustively identified male pheromonal compounds in house mice, two sesquiterpenes from the preputial gland, (i.e.,) E-b-farnesene and E, E-a-farnesene, are excreted in the urine in order to communicate information about sex and dominance. In addition, these compounds also influence on estrus induction in grouped females ${ }^{11}$. Therefore, they would seem to carry information that leads to aggression when perceived by male mouse and to an attraction when perceived by females ${ }^{12}$. Farnesol has been identified as the major volatile compound in the preputial gland of albino rat ${ }^{10,13}$ and detected the occurrence of $\alpha 2 \mathrm{u}$ with its bound putative pheromones (i.e., Farnesol1 and 2) in the preputial gland of house rat ${ }^{14}$. The pheromonal compound farnesol identified in the preputial gland of a rat is found to attract the opposite sex very efficiently ${ }^{15}$. We have also recently demonstrated through calcium image analysis that an extract
} 
a

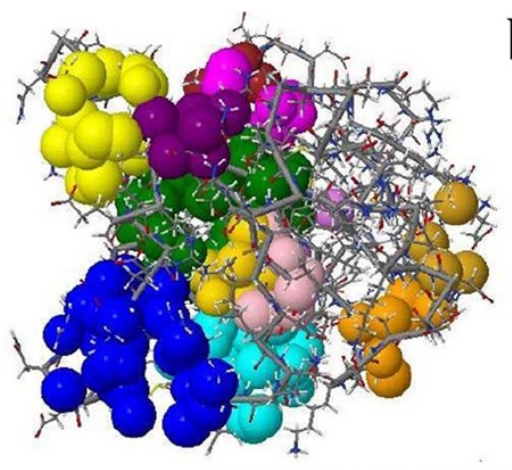

b

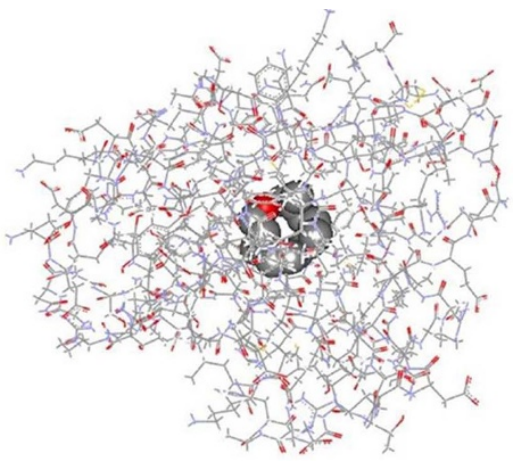

c

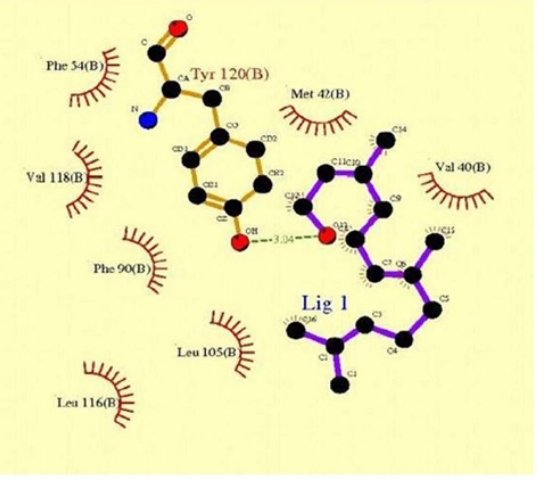

Figure $1 \mid$ Binding sites interaction was predicted using docking study in $\alpha 2 \mathrm{u}$. (a) The binding sites of the $\alpha 2 \mathrm{u}$ colored by several indications. (b) Docking study of $\alpha 2 \mathrm{u}$ with the farnesol. (c) The Ligplot analysis for protein and ligand interactions.

of rat preputial gland is capable of activating the olfactory receptor neuron (ORN), confirming that preputial gland and so as expound with farnesol has shown to be a potent source of pheromone property $^{16}$.

Bocskei et al. ${ }^{3}$ have reported the X-ray structure of a monoclinic crystal form of $\alpha 2 \mathrm{u}$ (Protein data bank code [PDB]: $2 \mathrm{a} 2 \mathrm{u}$ ). The protein is a member of the lipocalin superfamily which is distinguished by an unusual tertiary structure motif that features an eight-stranded antiparallel $\beta$-barrel shape forming a hydrophobic pocket in the protein ${ }^{17}$. Many of these proteins, such as the retinol binding proteins, the odorant-binding proteins ${ }^{18}$, the major urinary protein (MUP) ${ }^{19}$ and the retinol epididymal acid binding protein ${ }^{20}$ contain a hydrophobic-binding site for small lipophilic ligands. It is significant to note that the mouse MUP which is identical to $\alpha 2 \mathrm{u}$, binds with the major signaling ligands such as 2-sec-butyl-4,5-dihydrothiazole and 3,4-dehydro-exo-brevicomin ${ }^{21}$. Subsequently, it is demonstrated that the fluorescent molecule 2-naphthol binds to the 2-Isobutyl-3-methoxypyrazine (iBMP) binding site of major urinary proteins with strong affinity ${ }^{22}$. Overall, these proteins can react with various volatile constituents of the male mouse urine and induce puberty acceleration in female ${ }^{23}$. In common house rat (Rattus rattus), the $\alpha 2 \mathrm{u}$ also can binds with urinary pheromone compound in which 1-chlorodecane, a sex attractant compound, has been detected as the predominant bound compound ${ }^{15}$. The size of the ligand and its degree of flexibility can have a considerable influence on the ligand binding success ${ }^{24}$. The binding efficiency of $\alpha 2 \mathrm{u}$ and its interaction with the natural ligand are not yet investigated.

In the study of macromolecules, an in silico approach like molecular docking may through more light on the molecular interaction between $\alpha 2 \mathrm{u}$ with its natural ligand and the binding affinity score, interacting residues, intermolecular distance analysis which would ultimately lead to a better understating of receptor-ligand interaction. In support of such bioinformatics approach, fluorescence measurements would provide information about emission, excitation state and dissociation constant for bound $\alpha 2 \mathrm{u}$ with the fluorescent probe (2-naphthol). The study of $\alpha 2 \mathrm{u}$ represents photo-physical properties of fluorescence molecules in a hydrophobic environment. Hence in the present study, fluorescence emission spectrum of 2-naphthol characterized by the two major states (emission and excitation) was fully exploited at buffer $\mathrm{pH}$ 7.2.

Therefore, it is important to evaluate the interaction between farnesol and 2-naphthol for $\alpha 2 \mathrm{u}$ in order to obtain the binding affinity through docking analysis. Further, it is also necessary to investigate the binding affinity of 2 -naphthol with bound $\alpha 2 \mathrm{u}$ by fluorescence titration assay. The long-term objective is that the assessment of $\alpha 2 \mathrm{u}$ and molecular interaction with ligand would enhance the longevity of $\alpha 2 \mathrm{u}$ by protein engineering tool.

\section{Results}

Computational analysis. Computed atlas of surface topography of proteins analysis (CASTp). CASTp is a program performs automatic searching and measuring the surface binding pockets of desired proteins. This program was used to identify thirteen possible binding pockets in $\alpha 2 \mathrm{u}$ (Fig. 1a). The identified $\alpha 2 \mathrm{u}$ pockets are extremely hydrophobic, with only a handful of polar atoms at the surface of the pocket. It includes a ten-stranded antiparallel $\beta$-sheet, which forms an eight-stranded $\beta$-barrel structure with both $\mathrm{N}$ terminal and C-terminal extensions. Furthermore, the cavity within $\alpha 2 \mathrm{u}$ is roughly spherical in shape. Pocket one; being the largest is shown with green colour (Fig. 1a) contains the most residues (Table 1). Hence, our docking study focused on Pocket 1 of the chain B of $2 \mathrm{a} 2 \mathrm{u}$.

Molecular docking. We first docked farnesol with Pocket one of the protein shown as compound one in Fig. 1b. The conformation of the docked farnesol with the best fitness score was displayed as a balland-stick model. The detailed binding mode of farnesol with the protein distal pocket was composed by a number of hydrophobic residues including Val40, Met42, Phe54, Leu105, Phe90, Leu116, Val118 and Tyr120 (Fig. 1c). The docking study of the proteinligand complex, when clustering of the conformations was 
Table 1 | CASTp analysis confirmed of $\alpha_{2 u}$ - globulin binding pockets. The table shows the area and volumes of the 13 pockets found by CASTp. Locations of pockets with significant volumes are indicated

\begin{tabular}{|c|c|c|c|c|}
\hline aPocket & ${ }^{\mathrm{b}}$ Area,,$\AA^{2}$ & 'Volume, $\AA^{3}$ & ${ }^{\mathrm{d}}$ Color & ${ }^{\mathrm{e}}$ Amino acid residues with position \\
\hline 1 & 361.7 & 461 & green & $\begin{array}{l}\text { Met38, Val40, Met42, Phe54,Phe56, Leu69, Ala71, Val82, Tyr84, } \\
\text { Asn88, phe90, Phe103, Leu105, Met1 17,Val1 18, Tyr120 }\end{array}$ \\
\hline 2 & 214 & 185.6 & blue & $\begin{array}{l}\text { Glu34, Asn35, Gly36, Arg39, Phe4 1, Ile58, Lys59, Gly62, } \\
\text { Thr 154,Asp155 }\end{array}$ \\
\hline 3 & 127.1 & 23.8 & cyan & Phe20, Ser21, Phe41, Met42, Gln43, Arg57, Arg156, Leu 158 \\
\hline 4 & 92.7 & 91.3 & yellow & Lys28, Lys31, Asp85, Asn 107, Lys 109, Glu 1 12, Thr 1 13, Phe 114 \\
\hline 5 & 40.1 & 33.4 & magenta & Thr89, Phe90, Thr91, His 104, lle 106 \\
\hline 9 & 25.5 & 13.9 & brown & Thr74, Glu79, Glu79, Tyr80, Thr89 \\
\hline 10 & 39.2 & 22.3 & gold & Ser21, Val24, Val40, Val 118, Tyr 120 \\
\hline 11 & 32.4 & 17.1 & violet & Leu 15, Ile45, Leu52, Phe03 \\
\hline 12 & 26.4 & 12.5 & hotpink & His44, Asp46, Gly53, Tyr68 \\
\hline 13 & 10.8 & 6.8 & goldenrod & Arg99, Tyr 100, Ile 130, Lys 133 \\
\hline
\end{tabular}

performed, gave a low binding energy of $-6.42 \mathrm{kcal} / \mathrm{mol}$ with a Cluster RMS score value of 1.26. A hydrogen bond interaction could be observed between farnesol and hydroxyl group of TYR120 (Fig. 2a). Also, we docked 2-naphthol with $\alpha 2 \mathrm{u}$; the conformation of docked 2-naphthol got almost equal to farnesol. Indeed the 2naphthol had $-6.04 \mathrm{kcal} / \mathrm{mol}$; a more efficient hydrogen bond interaction occurred between 2-naphthol with TYR120 residue of $\alpha 2 \mathrm{u}$ (Fig. 2b). Since, the preliminary computational results provided a strong binding interaction between $\alpha 2 \mathrm{u}$ with farnesol and 2-naphthol. Further the experimental study is required to confirm the findings of this study.

Experimental analysis. Protein Purification. The 12\% sodium dodecyl sulphate- polyacrylamide gel electrophoresis (SDS-PAGE) was used to analyze the purity of the glandular protein. The rat preputial gland protein profiles revealed several proteins, in which low molecular mass of $18.54 \mathrm{kDa}$ proteins was expressed predominantly and the molecular mass of the $\alpha 2 \mathrm{u}$ commensurate with that of deduced PBPs (Fig. 3a). Purified protein was used for further proteomics analysis.

MALDI-TOF/MS experiment. The purified protein appeared as a single band with a low molecular mass of $18.54 \mathrm{kDa}$. The highly expressed $\alpha 2 \mathrm{u}$ proteins were excised and subjected to in-gel tryptic digestion. The high-quality of MALDI spectra was obtained for the $18.54 \mathrm{kDa}$ protein (Fig. $3 \mathrm{~b}$ ); the observed and expected masses of spectrum are shown in Table 2 and the protein were determined as $\alpha 2 \mathrm{u}$ by MALDI-TOF/MS. The $\alpha 2 \mathrm{u}$ was confirmed by the maximum resemblance of this protein (accession number: Q9JJI2) with 50\% sequence coverage (Fig. 3c).

Fluorescence titration study. The emission and excitation states appeared in fluorescence titration assay in different concentration of protein ligand fluorescence titration. Two types of titration experiments (Inversed and Direct) were used to measure the concentration of the protein ligand fluorescence. The inverse titration meant a presence of standard ligand concentration to measure the function of protein concentration in the ligand fluorescence. The direct titration defines a standard protein concentration used to measure the various functions of ligand concentration. Both titrations exhibited emission, excitation peaks at $350 \mathrm{~nm}$ and $420 \mathrm{~nm}$ respectively. In the fluorescence titration study, the fluorescence intensity was measured in different concentrations of $\alpha 2 \mathrm{u}$ (Viz., $2 \mu \mathrm{M}, 6 \mu \mathrm{M}$ and $15 \mu \mathrm{M}$ ) against standard $25 \mu \mathrm{M} 2$-naphthol. The $\alpha 2 \mathrm{u}$ concentration level decreased in inverse titration. The fluorescence emission also decreased at $360 \mathrm{~nm}$ and this was accompanied by an increase in fluorescence excitation at $420 \mathrm{~nm}$ (Fig. 4a). During direct titration, the fluorescence intensity was analyzed in different concentrations (Viz., $1 \mu \mathrm{m}, 3 \mu \mathrm{M}, 6 \mu \mathrm{M}$ and15 $\mu \mathrm{M}$ ) of 2-naphthol with standard $24 \mu \mathrm{M} \alpha 2 \mathrm{u}$. The undissociated event was noticed in the emission

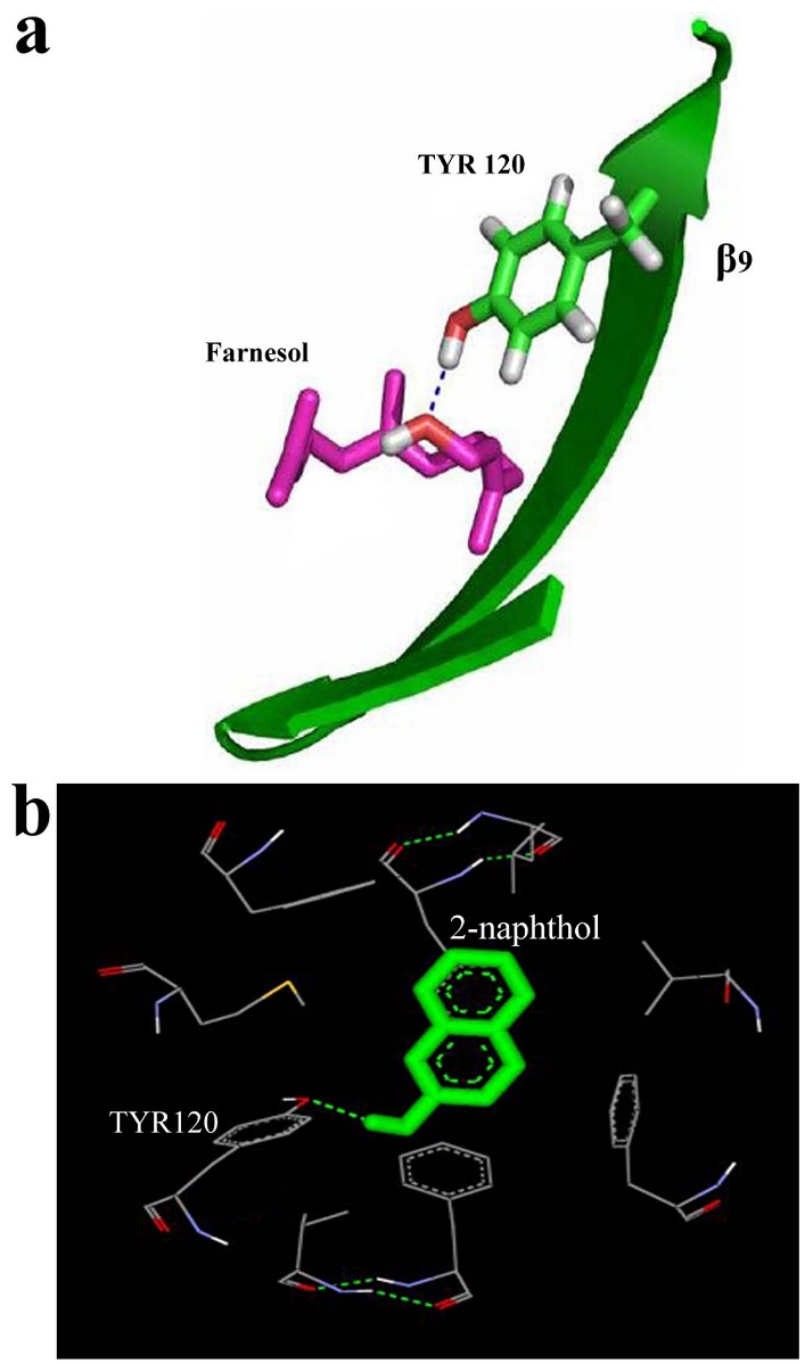

Figure $2 \mid$ Hydrogen bond interactions of farnesol and 2-naphthol are towards $\boldsymbol{\alpha} 2 \mathrm{u}$. (a) The location of the pheromone (farnesol) within the $\beta$ barrel suggests that the $\beta_{9}$ intervening loop may function as an entrance to the ligand binding site at TYR120. (b) 2-naphthol interaction with the same binding pocket of $\alpha 2 \mathrm{u}$ at TYR120. 


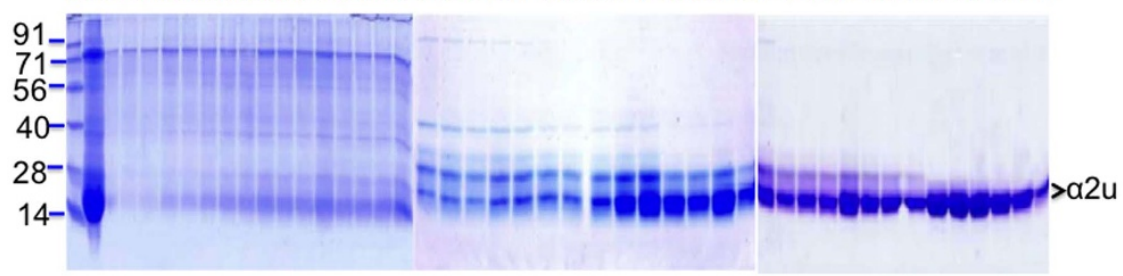

b

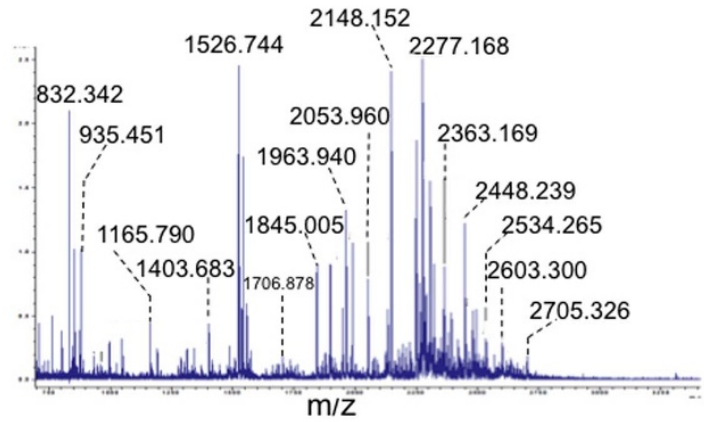

C

1 MKLLLLLLCL GLTLVCGHEE EASFERGNVD VDKLNGDWFS IVMASDKREK

51 IEENGSMRVF VQHIDVLENS LGFKFRIKVN GKCRELYLVA YKTPKDGEYF 101 VEYDGGNTFN ILKTDYDRYV MFHLVNFKDG ETFQLMNLFG RTKDLSSDIK 151 EKFAKLCVAH GITRENIIDV TKTNRCLQAQ G

Sequence Coverage: $\mathbf{5 0 \%}$

Matched peptides shown in Bold Red

Figure 3 Proteomics analysis of preputial gland of Indian commensal rat: (a) Proteins were extracted from the preputial gland and $40 \mu \mathrm{g}$ of protein was separated with the aid of $12 \%$ SDS-PAGE and the total band from the whole cell extract was subjected into gel filtration chromatography (GFC) lanes from 33 to 76 shows the fraction of protein lysates from GFC. The pure form of $\alpha 2 \mathrm{u}$ eluted from 72 to 76 fractions. (b) MALDI-TOF/MS spectra showed lowed molecular mass protein resemblance to $\alpha 2 \mathrm{u}$. (c) The matched peptides are shown as underlined and sequence coverage $50 \%$ of $\alpha 2 \mathrm{u}$ based on the database analysis.

state at $360 \mathrm{~nm}$ and the molecules dissociated into the excited state at $420 \mathrm{~nm}$. The fluorescence intensity was vertically increased in the initial part of the titration at $350 \mathrm{~nm}$, while was absent at $420 \mathrm{~nm}$ (Fig. 4b). In addition, the fluorescence intensity ratio F/F0 measured (F means fluorescence intensity in the presence of farnesol and F0 represents the fluorescence intensity in the absence of farnesol) ${ }^{22}$. The results revealed that, there is an affinity between $\alpha 2 \mathrm{u}$ and 2naphthol as determined through the fluorescence titration confirming the binding of 2-naphthol in the same active sites of natural endogenous ligand. Therefore, 2-naphthol was displaced from the binding cavity of bound $\alpha 2 \mathrm{u}$ while increasing the protein concentration at $360 \mathrm{~nm}$ in inverse titration.
Stern-volmer plot using KI quenching. The quenching experiments was applied to analyze the fluorescence intensity of bound $\alpha 2 \mathrm{u}$ with 2-naphthol in emission excited state at $350 \mathrm{~nm}$ and $420 \mathrm{~nm}$ respectively. The potassium iodide (KI), quenching experiments, showed that the quenching became prominently more effective during concentration increased through Stern-Volmer plot. During, Free 2-naphthol emission peak and emission, an excitation peak was observed for bound $\alpha 2 \mathrm{u}$ with 2-naphthol. An emission peak represented a dynamic quenching and it was decreased during increasing concentration of the quencher at $350 \mathrm{~nm}$ based on inverse titration; the emission peak of Free 2-naphthol increases while increasing quencher at $350 \mathrm{~nm}$ (Fig. 4c). Hence, the

Table 2 | Tryptic digested $18.54 \mathrm{kDa}(\alpha 2 \mathrm{u})$ : The observed and expected masses $[\mathrm{M}+\mathrm{H}]^{+}$of $\alpha 2 \mathrm{u}$ by mass spectroscopy

\begin{tabular}{lrccl} 
Start & End & Observed masses $(\mathrm{m} / \mathrm{z})$ & Expected masses $(\mathrm{m} / \mathrm{z})$ & \multicolumn{1}{c}{ Sequence } \\
\hline 34 & 48 & 1738.84 & 1737.83 & K.LNGDWFSIVMASDKR.E \\
49 & 58 & 1192.56 & 1191.56 & R.EKIEENGSMR.V \\
51 & 58 & 935.45 & 934.44 & K.IEENGSMR.V \\
59 & 74 & 1844.99 & 1843.99 & R.VFVQHIDVLNSLGFK.F \\
59 & 76 & 2148.14 & 2147.13 & R.VFVQHIDVLENSLGFKFR.I \\
85 & 92 & 1947.53 & 998.52 & R.ELYLVAYK.T \\
114 & 128 & 1526.73 & 1946.92 & K.TDYDRYVMFHLVNFK.D \\
129 & 141 & 1373.69 & 1525.73 & K.DGETFQLMNLFGR.T \\
153 & 164 & 1026.56 & 1372.69 & K.FAKLCVAHGITR.E \\
156 & 164 & & 1025.55 & K.LCVAHGITR.E
\end{tabular}



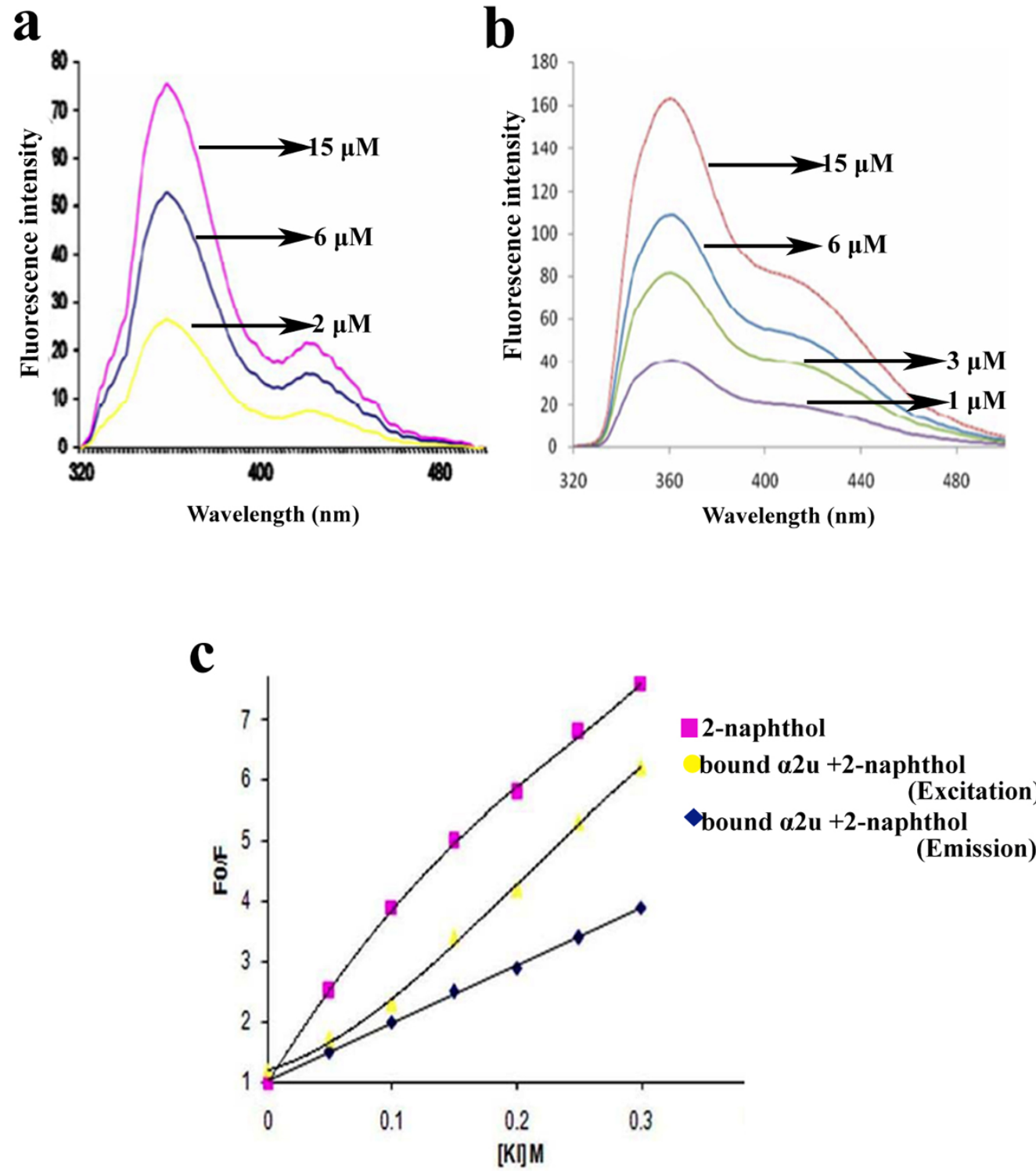

Figure $4 \mid$ Fluorescence titrations study. (a) The inverse titration experiments showed spectra of $25 \mu \mathrm{M} 2$-naphthol at different $\alpha 2 \mathrm{u}$ concentrations shows $2 \mu \mathrm{M}$ (yellow line), $6 \mu \mathrm{M}$ (blue line), and $15 \mu \mathrm{M}$ (pink line). (b) The direct titration experiments show spectra of $24 \mu \mathrm{M} \alpha 2 \mathrm{u}$ at different 2 naphthol increasing concentrations $(1 \mu \mathrm{M}, 3 \mu \mathrm{M}, 6 \mu \mathrm{M}$ and $15 \mu \mathrm{M})$. (c) Stern-Volmer plots of 2-naphthol with potassium iodide (KI) fluorescence quenching experiments.

maximum displacement of 2-naphthol was noticed instead of farnesol occupied the $\alpha 2 \mathrm{u}$ binding sites.

\section{Discussion}

In molecular docking study, $\alpha 2 \mathrm{u}$ has the largest pocket on a protein that has the most likely active site ${ }^{25}$. It exists inside the protein and occupies an area of $361.7 \AA^{2}$ and a volume of $461 \AA^{3}$. The side chains $\mathrm{Val}$ and Tyr account for the hydrophobic nature of this pocket, while the hydroxyl groups from a Ser residue contributes to its hydrophobicity. All the listed amino acids with the corresponding residue number (Table 1) and the detailed binding sites of $\alpha 2 \mathrm{u}$ have been depicted (Fig. 1a).

During the docking process it was observed that the farnesol docked to $\alpha 2 \mathrm{u}$ with the lowest energy. The occurrence of lowest energy within the protein-ligand complex is mainly due to stabilization by maximum hydrogen bonding interactions (Fig. 1b). Proteinligand interaction calculated by Ligplot program showed interacting residues in the docking process. The recognition and affinity of protein towards the ligand were interpreted based on the atomic distances lesser than $3.04 \AA$, implying that the interactions between the protein and ligand occur only within the active site pocket. A hydrogen bonding interaction could be observed between the ligand and the hydroxyl group of TYR120 (Fig. 1c). However, this can only function as a weak hydrogen-bond acceptor and would be able to interact only with the open diol form of the ligand, which is not very physiologically relevant. The $\beta$-barrel structure encloses a cup like structure and allows the non-covalent binding of volatile semio chemicals; these properties have given rise to $\mathrm{PBP}$ or Pheromone Carrying Proteins (PCPs) ${ }^{21,26}$. The characterizations of glandular $\alpha 2 \mathrm{u}$ observed in this study are consistent with previous reports on urinary PCPs in male rats and mice acting as pheromone carriers ${ }^{2,15}$. The location of farnesol within the $\beta$-barrel suggests that the $\beta_{9}$ intervening loop may function as an entrance to the ligand-binding site (Fig. 2a). Further it is important to note that the 2-naphthol interacts with the same binding pocket residue of TYR 120 as that of farnesol (Fig. 2b). A previous report proposed that the loop a/b function as the entrance to the interior of the $\beta$-barrel ${ }^{27}$. 
After generating results from computational analysis, the outcome was extrapolated and confirmed by an in vitro study. The analysis of SDS-PAGE protein profiles also revealed that the rat preputial gland comprised $18.5 \mathrm{kDa}$ band from lane $72-76^{\text {th }}$ fractions (Fig. 3a). These results are consistent with the previous reports on the presence of $17 \mathrm{kDa}$ lipocalin protein in male urine of mouse and the X-ray crystal structure form of $\alpha 2 \mathrm{u}$ at $2.5 \AA$ resolution ${ }^{3}$. In the present study, the presence of $18.54 \mathrm{kDa}$ protein was confirmed as $\alpha 2 \mathrm{u}$ with the aid of MALDI-TOF/MS (Fig. 3b). The spectra represented monoisotopic masses that were pooled together and further analyzed with MASCOT search ${ }^{28}$. The results showed the highest resemblance to $\alpha 2 \mathrm{u}$ (accession number: Q9JJI2), and provide $50 \%$ of sequence coverage (Fig. 3c). The matched and expected mass values of peptides are listed (Table 2). Subsequently, the fluorescence titration analysis provided an adequate evidence to understand the binding efficiency of $\alpha 2 \mathrm{u}$ with farnesol and 2-naphthol. By inverse titration, the presence of 2-naphthol with $2 \mu \mathrm{M}, 6 \mu \mathrm{M}$ and $15 \mu \mathrm{M}$ was found to increase the concentration of $\alpha 2 \mathrm{u}$ fluorescence on emission at $350 \mathrm{~nm}$. On the other hand, to the presence of $\alpha 2 \mathrm{u}$ at $420 \mathrm{~nm}$ on excitation state the 2-naphthol was dissociated, that would represent to originate only from the unbound fluorescent molecules remaining in solution (Fig. 4a). In contrast to direct titration, the equivalent fluorescence intensity emission of 2-naphthol increased at $360 \mathrm{~nm}$ and decreased the level of concentration, as well as dissociated event occurred at $420 \mathrm{~nm}$ (Fig. 4b). Hence, the hypothesis put forward is that the 2-naphthol is displaced from the protein binding site by farnesol.

In order to identify the interaction of 2-naphthol with bound $\alpha 2 \mathrm{u}$, fluorescence quenching experiments were carried out using potassium iodide as quencher. The fluorescence intensity decreases with an increase the concentration of the quencher. Based on the inverse titration, the fluorescence emission between the quencher and bound $\alpha 2 \mathrm{u}$ with 2-naphthol showed a linear and non-linear plots through Stern-Volmer method (Fig. 4c). In addition, the maximum emission for the calculated fluorescence spectrum of the bound species is blueshifted and more intense ${ }^{29}$. The dissociation constant of $\alpha 2 \mathrm{u}$ with 2naphthol was measured as $k d=0.46 \mu \mathrm{M}$. Hence, we presume through the docking study that 2-naphthol interact same binding pocket as that of farnesol. It may be postulated that the farnesol might possess the same $k d$ as that of 2-naphthol. This is the first report in reference to the target of farnesol and 2-naphthol is interacting on the same binding pockets of $\alpha 2 \mathrm{u}$. Since, the farnesol has a good binding efficiency with $\alpha 2 \mathrm{u}$. It can be considered as an ideal candidate in the development of a pheromone trap or biotrap for the rodent pest management program. Finally, we found trends in the level of clustering and predicted binding energies that are able to distinguish natural volatile ligands from non-binding molecules. This approach would be useful to design ligand based engineered protein active sites that can be created and in designing the biotrap based on pheromone compounds for rodent pest control programs.

\section{Methods}

Molecular structure and optimization. The 3D structure of $\alpha 2 \mathrm{u}$ B chain (PDB code: $2 \mathrm{a} 2 \mathrm{u}$ ) was obtained from the RCSB-PDB (http://www.rcsb.org/pdb). The crystal structure of the protein deposited in the PDB was recorded at a resolution of $2.5 \AA$. The chemical compound farnesol has been detected in the preputial gland of the Indian common house rat by gas chromatography coupled to mass spectrometry. The 2D structure of the farnesol was obtained from the Pubchem compound module (http://pubchem.ncbi.nlm.nih.gov/) and the compound id (CID) are 1549109 (farnesol) and 8663 (2-naphthol). The AMBER force field and Gasteiger charges were assigned and fixed successfully for farnesol using VEGA ZZ software ${ }^{30}$. The 2D structure of the compound was then converted into the corresponding $3 \mathrm{D}$ coordinates using the Babel server (http://openbabel.sf.net).

Molecular docking study. The active site of $\alpha 2 \mathrm{u}$ was analyzed using the CASTp program to identify and characterize the surface accessible pockets of the protein. The pockets were characterized by two parameters, namely the solvent accessible surface (SA, Richards' surface) and the molecular surface (MS, Connolly's surface) ${ }^{31}$. The AutoDock4 program used to calculate the interaction between the farnesol and $\alpha 2 \mathrm{u}$ using the lamarckian genetic algorithm (LGA). The purpose to calculate the possible conformations for farnesol and 2-naphthol as it binds to the protein ${ }^{32}$. During docking analysis, a maximum of ten best conformers with lowest binding energy were considered for farnesol. All calculations were performed on an SGI FUEL

workstation. The resulting structural files were visualized and analyzed using Pymol software and the LIGPLOT 4.2.2 program.

Experimental animals. Male house rats (Rattus rattus) were collected from the nearby village of Bharathidasan University and were acclimatized to laboratory condition for 2 weeks prior to experiments. They were housed in a cage, in the laboratory, reared on pelleted food (Sai Durag Ltd, Bangalore) and water ad libitum. Male and female rats were housed separately in poly propylene cages $(40 \times 25 \times$ $15 \mathrm{~cm}$ ) with $2 \mathrm{~cm}$ of rice husk lining the bottom as bedding material. The bedding material was changed once in 3 or 4 days to maintain the hygienic condition of the animals. All animals were maintained in L \& D cycle (12:12) throughout the experimental period. The procedures were strictly adhered to following the guidelines for animal care by Institutional Animal Ethics Committee (IAEC), India.

Preparation of preputial gland extract. The sexually matured and reproductively active normal male house rats (Rattus rattus) were used for the collection of samples and animals were sacrificed through cervical dislocation and preputial glands were collected. The glands were then homogenized with the PBS (pH 7.2) and centrifuged at $4^{\circ} \mathrm{C}$ for $15 \mathrm{~min}$ at $10,000 \mathrm{rpm}$. After centrifugation, the clear supernatant was collected and separated by size exclusion chromatography.

Protein purification by size exclusion chromatography. The $\alpha 2 \mathrm{u}$ was identified and purified from preputial gland of Rattus rattus. Protein purified by Sephadex G-50 (gel-filtration chromatography). The column (length: $28 \mathrm{~cm}$; i.d.: 0.7 ) was packed with Sephadex G-50, and equilibrated with $10 \mathrm{mM}$ phosphate buffer ( $\mathrm{pH}$ 7.8). After the equilibration, the concentrated glandular homogenates were poured onto the top of the column in a single motion and eluted with the same buffer at a flow rate of $0.5 \mathrm{~mL} / \mathrm{h}$. The fractions were analyzed for optical absorbance at $280 \mathrm{~nm}$ with a UVvisible spectrophotometer and the purity was checked once again by $12 \%$ SDS-PAGE

SDS-PAGE. The $12 \%$ of SDS-PAGE carried out using a slightly modified protocol ${ }^{33}$ with $30 \mu \mathrm{L}$ of protein in each fraction; $4 \mu \mathrm{L}$ of medium range molecular weight marker (Bangalore Genie cat. No. PMW-M) was used for the molecular weight reference. The entire electrophoresis was carried out at a constant voltage (50 V) and at room temperature for 4 hours and bands was detected by Coomassie brilliant blue stain.

Mass spectrometry analysis. $\alpha 2 \mathrm{u}$ was prepared by the tryptic in-gel digestion and MALDI-TOF/MS methods according to Muthukumar et al. ${ }^{34}$. The collected mass spectra were processed with FLEX analysis software; the monoisotopic peptide masses were assigned and used in the database search. The protein identification was accomplished utilizing the MASCOT search (http//www.matrixscience.com). Scores $>65$ were considered to be significant $(\mathrm{p}<0.05)$ in the MASCOT search.

Fluorescence study with titrations. For fluorescence study, 2-naphthol was used without further purification methods and chemical obtained from Sigma (St. Louis, MO, USA). The $\alpha 2 \mathrm{u}$ protein was purified by chromatography method ${ }^{15}$ and the determination of concentration was calculated through spectrophotometrically using the molar extinction coefficient $€_{276}=11450 \mathrm{~cm}^{-1} \mathrm{M}^{-1}$ at $276 \mathrm{~nm}^{24}$. The following concentrations of purified protein $(24 \mu \mathrm{M})$, and 2-naphthol ( $1 \mathrm{mM}$ stock) was prepared in ice-cold $100 \%$ ethanol and they were stored in the dark at $4{ }^{\circ} \mathrm{C}$, and the final concentration of $25 \mu \mathrm{M}$ diluted for each experiment. The $\mathrm{pH} 7.2$ for $10 \mathrm{mM}$ Tris and room temperature maintained in all experiments. Fluorescence measurements were carried out on an FP-6500 spectrofluorimeter (JASCO, Japan) equipped with a thermostat bath and $1.0 \mathrm{~cm}$ quartz cells, using $5 \mathrm{~nm} / 5 \mathrm{~nm}$ slit widths. All pH values were measured by a $\mathrm{pH}-3 \mathrm{c}$ digital $\mathrm{pH}$-meter (Shanghai Lei Ci Device Works, Shanghai, China) with a combined glass-calomel electrode. We used $25 \mu \mathrm{M} 2$ naphthol, and $24 \mu \mathrm{M} \alpha 2 \mathrm{u}$ in a cuvette and then consecutively diluted by adding aliquots of a $25 \mu \mathrm{M}$ of 2-naphthol for the inverse titration. Instead of 2-naphthol, buffer was prepared as a blank and added $3 \mathrm{ml}$ of $24 \mu \mathrm{M} \alpha 2 \mathrm{u}$ solution in a cuvette and aliquots of $1 \mu \mathrm{M} 2$-naphthol stock solution was added in the direct titration.

Fluorescence quenching experiment. Our previous study shows the bound form of farnesol with purified $\alpha 2 \mathrm{u}$, therefore, we were interested to explore the binding efficiency of farnesol with $\alpha 2 \mathrm{u}$. Fluorescence quenching analysis was applied using $2 \mathrm{M}$ stock solution of potassium iodide (KI). The KI acts as a quencher and the fresh solution were prepared for the experiment.

1. Petrulis, A. Chemosignals, hormones and mammalian reproduction. Horm. Behav. 63, 723-741 (2013).

2. Cavaggioni, A. \& Mucignat-Caretta, C. Major urinary proteins, alpha (2U)globulins and aphrodisin. Biochim. Biophys. Acta. 1482, 218-228 (2000).

3. Bocskei, Z. et al. Pheromone binding to two rodent urinary proteins revealed by Xray crystallography. Nature. 360, 186-188 (1992).

4. Sankar, R. \& Archunan, G. Identification of putative pheromones in bovine (Bos taurus) faeces in relation to estrus detection. Anim. Reprod. Sci. 103, 149-153 (2008). 
5. Rajanarayanan, S. \& Archunan, G. Occurrence of flehmen in male buffaloes (Bubalus bubalis) with special reference to estrus. Theriogenology 61, 861-866 (2004).

6. Grammer, K., Fink, B. \& Neave, N. Human pheromones and sexual attraction. Eur. J. Obstet. Gynecol. Reprod. Biol. 118, 135-142 (2005).

7. Rekwot, P. I., Ogwu, D., Oyedipe, E. O. \& Sekoni, V. O. The role of pheromones and biostimulation in animal reproduction. Anim. Reprod. Sci. 65, 157-170 (2001).

8. Leon, M. [Chemical Communication in Mother Young Interactions] Pheromone and Reproduction in Mammals. [Vandenbergh, J. G. (ed.)] [39-77] (Academic Press, New York, 1983).

9. Agosta, W. C. Chemical Communication: The Language of Pheremones. [Sheila S Reilly (ed.)] [23-25] (Scientific American Library, New York, 1992).

10. Ponmanickam, P. et al. Identification of testosterone-dependent volatile compounds and proteins in the preputial gland of rat Rattus norvegicus. Gen. Comp. Endocrinol. 167, 35-43 (2010).

11. Ma, W., Miao, Z. \& Novotny, M. V. Induction of estrus in grouped female mice (Mus domesticus) by synthetic analogues of preputial gland constituents. Chem. Senses. 24, 289-293 (1999).

12. Bronson, F. H. \& Caroom, D. Preputial gland of the male mouse; attractant function. J. Reprod. Fert. 25, 279-282 (1971).

13. Kannan, S., Ramesh Kumar, K. \& Archunan, G. Sex attractants in male preputial gland: Chemical identification and their role in reproductive behaviour of rats. Curr. Sci. 74, 689-691 (1998).

14. Rajkumar, R. et al. Detection of $\alpha 2 \mathrm{u}$-Globulin and its bound putative pheromones in the preputial gland of indian commensal rat (Rattus rattus): using mass spectrometry. Rapid Commun. Mass. Spectrom. 24, 721-728 (2010).

15. Liebich, H. M., Zlatkis, A., Bertsch, W., Van Dahm, R. \& Whitten, W. K. Identification of dihydrothiazoles in urine of male mice. Biomed. Mass Spectrom. 4, 69-72 (1977).

16. Ponmanickam, P. et al. Preputial gland activates olfactory receptor neurons in rat: Calcium imaging study using laser scanning confocal microscopy. Indian J. Biochem. Biophy. 50, 242-246 (2013).

17. Papiz, M. Z. et al. The structure of beta-lactoglobulin and its similarity to plasma retinol- binding protein. Nature 324, 383-385 (1986).

18. Bianchet, M. A. The three-dimensional structure of bovine odorant binding protein and its mechanism of odor recognition. Nat. Struct. Biol. 3, 934-949 (1996).

19. Rajkumar, R., Prakash, S., Archunan, G. \& Sowdhamini, R. Primary structural documentation of the major urinary protein of the Indian commersal rat (Rattus rattus): using proteomic plotform. Protein Pept. Letters 17, 449-457 (2010).

20. Newcomer, M. E. Structure of the epididymal retinoic acid binding protein at 2.1 A resolution. Structure 15, 7-18 (1993).

21. Bacchini, A., Gaetani, E. \& Cavaggioni, A. Pheromone binding proteins of the mouse, Mus musculus. Experientia. 48, 419-421 (1992).

22. Sartor, G. et al. Determining the binding capability of the mouse major urinary proteins using 2-naphthol as a fluorescent probe. Anal Biochem. 292, 69-75 (2001).

23. Novotny, M. V. et al. A unique urinary constituent, 6-hydroxy-6-methyl-3heptanone, is a pheromone that accelerates puberty in female mice. Chem. Biol. 6 , 377-383 (1999).

24. Cavaggioni, A., Findlay, J. B. \& Tirindelli, R. Ligand binding characteristics of homologous rat and mouse urinary proteins and pyrazine-binding protein of calf. Comp. Biochem. Physiol. B. 96, 513-20 (1990).
25. Liang, J., Edelsbrunner, H., Fu, P., Sudhakar, P. V. \& Subramaniam, S. Analytical shape computing of macromolecules II: Identification and computation of inaccessible cavities inside proteins. Proteins 33, 18-29 (1998).

26. Briand, L., Trotier, D. \& Pernollet, J. C. Aphrodisin: an aprodisiac lipocalins secreted in hamster vaginal secretions. Peptides 25, 1545-1552 (2004).

27. Flower, D. R. A lipocalin protein family: A role in cell regulation. FEBS Lett. 354, 7-11 (1994).

28. Helsens, K., Martens, L., Vandekerckhove, J. \& Gevaert, K. MascotDatfile: An open-source library to fully parse and analyse MASCOT MS/MS search results. Proteomics 7, 364-366 (2007).

29. Lucke, C. et al. Solution structure of mouse recombinant major urinary protein. Eur. J. Biochem. 266, 1210-1218 (1999).

30. Pedretti, A., Villa, L. \& Vistoli, G. VEGA: A versatile program to convert, handle and visualize molecular structure on windows-based PCs. J. Mol. Graph. 21, 47-49 (2002)

31. Binkowski, T. A., Naghibzadeh, S. \& Liang, J. CASTp: Computed Atlas of Surface Topography of proteins. Nucleic Acids Res. 31, 3352-3355 (2003).

32. Morris, G. M., Goodsell, D. S., Huey, R. \& Olson, A. J. Distributed automated docking of flexible ligands to proteins: parallel applications of AutoDock 2.4. J. Comput. Aided Mol. Des. 10, 296-304 (1996).

33. Laemmli, U. K. Cleavage of structural proteins during the assembly of the head of bacteriophage T4. Nature 227, 680-685 (1970).

34. Muthukumar, S. et al. Urinary Lipocalin Protein in a Female Rodent with Correlation to Phases in the Estrous Cycle: An Experimental Study Accompanied by In Silico Analysis. PLoS ONE 8, e71357 (2013).

\section{Acknowledgments}

We thank Dr. Chellam Balasundram, UGC-Emeritus Professor, Bharathidasan University for critical reading of this manuscript. The facility availed through UGC, UGC-SAP and DST-PURSE, Government of India is acknowledged. PP acknowledges the support from the LKC School of Medicine, NTU Singapore.

\section{Author contributions}

R.I., R.R., P.P. and G.A. designed the study; R.I., R.R. and A.R.M. performed research; R.I., R.R., A.R.M., D.R. and P.P. analyzed data; R.I., R.R., P.P. and G.A. wrote the manuscript.

\section{Additional information}

Competing financial interests: The authors declare no competing financial interests.

How to cite this article: Ilayaraja, R. et al. Evaluating the binding efficiency of pheromone binding protein with its natural ligand using molecular docking and fluorescence analysis. Sci. Rep. 4, 5201; DOI:10.1038/srep05201 (2014).

This work is licensed under a Creative Commons Attribution-NonCommercialNoDerivs 3.0 Unported License. The images in this article are included in the article's Creative Commons license, unless indicated otherwise in the image credit; if the image is not included under the Creative Commons license, users will need to obtain permission from the license holder in order to reproduce the image. To view a copy of this license, visit http://creativecommons.org/licenses/by-nc-nd/3.0/ 